\title{
Evaluación de la calidad del servicio estudiantil en la carrera de Derecho de una Institución de Educación Superior pública de Guayaquil
}

\section{Evaluation of the student services quality in the Law Career from a Public Institution of Higher Education in Guayaquil}

\author{
María José Fuentes Durán ${ }^{1}$
}

\author{
Palabras clave: \\ Calidad de \\ servicio, \\ Satisfacción, \\ SERVPERF, \\ Deserción.
}

\begin{abstract}
Resumen
El presente estudio se realiza con la necesidad de evaluar la calidad del servicio estudiantil en la carrera de Derecho de una Institución de Educación Superior pública de Guayaquil, debido al aumento de la deserción universitaria en los últimos años en el Ecuador; el cual puede estar vinculado con la baja calidad del servicio estudiantil. Por tal motivo, se utilizó la herramienta SERVPERF para valorar el nivel de satisfacción y experiencia mediante la estimación de la tangibilidad, fiabilidad, capacidad de respuesta, seguridad y empatía. Concluyendo en que el modelo es aplicable para la evaluación de servicios estudiantiles en Instituciones de Educación superior, en el cual mediante un análisis convergente se logró visualizar que las dimensiones que tenían más efecto sobre la satisfacción fueron la Tangibilidad y la Empatía.
\end{abstract}

Códigos JEL: L89

\section{Keywords:}

Services Quality, Satisfaction, SERVPERF, Dropout.

\footnotetext{
${ }^{1}$ Universidad de Especialidades Espíritu Santo (Guayaquil-Ecuador).

E-mail: mariajosefuentes@uees.edu.ec

${ }^{1}$ Universidad de Guayaquil (Guayaquil-Ecuador).

E-mail: maria.fuentesdu@ug.edu.ec
} 


\section{INTRODUCCIÓN}

El progreso constante de la deserción universitaria en los últimos años en el Ecuador, ha sido un problema de gran relevancia para el Estado, donde según investigaciones, esta situación puede deberse a una falta de programas de apoyo académico, por particularidades del sistema educativo o por la baja calidad del servicio estudiantil (Verdesoto, Mora, y Torres, 2018). Un informe de la Secretaría Nacional de Educación Superior, Ciencia, Tecnología e Innovación (Senescyt), indica que de cerca de los 400.000 estudiantes que se encuentran aproximadamente en el sistema educativo, un $26 \%$ ha optado por la deserción de su carrera (Torres M. , 2019).

En las entidades públicas del Ecuador, es una constante el brindar un servicio al cliente que carece del mínimo deseable, haciendo énfasis en las instituciones de educación superior las cuales reciben miles de estudiantes nuevos semestralmente, donde lamentablemente se conoce que muchos estudiantes se quejan de los servicios que brindan estas instituciones; partiendo desde el ornato y las diferentes instalaciones de la misma hasta el trato que reciben los alumnos por parte de todos los administrativos indistintamente.

Es importante recordar que las Instituciones Educación Superior (IES) públicas, albergan a miles de estudiantes que serán el estandarte de estas instituciones, los mismos que pueden dar testimonio de la calidad del servicio de manera más fiable que cualquier noticiero o medio de información. Es por esto que lograr mejorar el servicio de estas organizaciones, es esencial no solo para la comunidad sino también para el bienestar del Estado, el cual es quien lo administra.

Dentro de este contexto, es necesario enfatizar que el servicio al cliente persigue la satisfacción del consumidor, brindándole apoyo, orientación e instrucciones respecto a lo que este necesite, por lo que las instituciones de educación superior no deben quedarse atrás en el cumplimiento básico del mismo (Lami, 2019). En el contexto cambiante de la actualidad en el mundo y aún más en la educación superior, los tecnológicos y las universidades terminan compitiendo para inscribir y retener a los estudiantes que están "comprando" o "pagando" por mejores instalaciones, mejores servicios, mejores planes de estudio, oportunidades curriculares y un mejor potencial para obtener un trabajo (todo a un precio reducido) (Bustamante, 2019).

Entonces partiendo desde ese principio, tecnológicos y universidades deben considerar cómo brindar un excelente servicio a los estudiantes; sin embargo, sigue habiendo una resistencia frecuente de considerar a los estudiantes universitarios como clientes (Peñalvo, Corell, Abella-García y Grande, 2020). Los estudiantes y los clientes no deben ser mutuamente excluyentes, ya que todas estas instituciones brindan un servicio educativo y sus estudiantes son los clientes que pueden expresar su satisfacción con el proceso por el que transitan al cursar sus años de estudio superior.

El alto grado de insatisfacción de parte de los estudiantes de las IES públicas es evidente en redes sociales $\mathrm{y}$ en todos los medios de información en los que se puede evidenciar denuncias hacia autoridades, docentes e incluso de las deficientes instalaciones de las mismas. La ineficiencia en la atención a los usuarios, edificios carecientes de mantenimiento y aseo y los recursos tecnológicos limitados son solo unos cuantos de los muchos problemas de servicios que acaecen a estas instituciones en el Ecuador. Particularmente en Guayaquil se encuentra una de las instituciones más grandes que por estos mismos motivos ha tenido que ser intervenida por el estado en varias ocasiones.

Por lo expuesto anteriormente, mediante el presente trabajo investigativo se plantea realizar un estudio que permita evidenciar que aspectos, mayormente administrativos, se podrían mejorar porque provocan un alto nivel de insatisfacción a los usuarios, refiriéndose tanto a estudiantes 
actuales como a egresados y graduados de la institución.

Varias investigaciones demuestran que los estudiantes satisfechos tienen más probabilidades de comprometerse y continuar sus estudios que los estudiantes insatisfechos, ya que es posible que estén menos dispuestos a asistir regularmente a clases y que posteriormente estos dejen sus estudios (Bernal, Lauretti, y Agreda, 2016). Dada la importancia de los niveles de satisfacción de los estudiantes en las instituciones de educación superior, ha habido un aumento en el interés en examinar los factores que afectan la satisfacción del alumnado.

El problema de la deserción estudiantil en la educación superior ha constituido un problema medular en los últimos años para las universidades latinoamericanas; donde algunas investigaciones han puesto en evidencia el porcentaje significativo de alumnos que no han podido culminar sus estudios, con las complicaciones en términos socioeconómicos que conlleva (Pérez et al, 2016). La deserción estudiantil es una situación recurrente en las IES públicas del Ecuador, donde si bien varios aspectos pueden ser considerados para tratar esta problemática, mediante el presente trabajo investigativo, será tratado bajo la perspectiva de la satisfacción estudiantil y el nivel experiencia que este puede tener dentro de las instalaciones.

Entrando es este contexto, en el estudio se plantea evaluar la calidad del servicio estudiantil en la carrera de Derecho de una Institución de Educación Superior pública de Guayaquil, utilizando la herramienta SERVPERF, el cual permite valorar el nivel de satisfacción y experiencia mediante la estimación de cinco dimensiones, los cuales son: elementos tangibles, fiabilidad, capacidad de respuesta, seguridad y empatía.

\section{MATERIALES Y MÉTODOS/ METODOLOGÍA}

Para el presente trabajo investigativo se plantea un enfoque cuantitativo del servicio actual que reciben los alumnos y las mejoras que ellos propongan para su implementación. En la metodología cuantitativa se recogen datos medibles y con base científica bajo los cuales se formulan interrogantes $y$ se empieza la recolección de estos datos para comprobar los mismos (Monje, 2011).

En la investigación cualitativa se capta la realidad social mediante la visión de aquellos quienes están siendo estudiados o analizados, basado en la percepción que tienen los sujetos de estudio. Busca conceptos sobre la realidad, pero en base a los comportamientos (Bracho, Fernández, \& Díaz, 2021).

El uso de una combinación de datos cualitativos y cuantitativos puede mejorar una evaluación al garantizar que las limitaciones de un tipo de datos se equilibren con las fortalezas de otro. Esto asegurará que se mejore la comprensión al integrar diferentes formas de conocimiento (Zacarías y Supo, 2020). La mayoría de las evaluaciones recopilarán tanto datos cuantitativos (números) como datos cualitativos (texto, imágenes) para integrar ambos conocimientos de manera que nos beneficie en la creación de nuestro plan estratégico (Caminotti y Toppi, 2020).

La población del presente trabajo investigativo está compuesta por los alumnos de la Carrera de Derecho de una IES pública de la ciudad de Guayaquil, que pertenece a la Facultad de Jurisprudencia Ciencias Sociales y Política; y está compuesta de la siguiente manera:

Tabla 1

Estudiantes de la Facultad de Jurisprudencia Ciencias Sociales y Política

\begin{tabular}{llllll}
\hline Carrera & Modalidad & Sistema & \multicolumn{2}{c}{ Estudiantes } \\
& & & Hombres & Mujeres & Total \\
\hline Derecho & Anual & Presencial & 1362 & 1454 & 2816 \\
Derecho & Semestral & Presencial & 740 & 855 & 1595 \\
Sociología & Semestral & Presencial & 179 & 231 & 500 \\
\hline
\end{tabular}

Fuente: IESS pública de la ciudad de Guayaquil (2016)

Realizando una segmentación de alumnos de la Carrera de Derecho, se establece 2816 personas en la modalidad anual y 1595 en semestral, 
estableciendo así una población de estudio de 4411 estudiantes.

Para el desarrollo del estudio se pretende implementar un método de muestro no probabilístico, por conveniencia, tomando de referencia a los estudiantes de la Carrera de Derecho de una IES pública de la ciudad de Guayaquil, que estén en la accesibilidad del investigador.

El muestro no probabilístico por conveniencia consistente en seleccionar una muestra de la población por el hecho de que sea accesible. Es decir, las personas empleadas en la investigación se seleccionan porque están fácilmente disponibles y no porque hayan sido seleccionados mediante un criterio estadístico (Sampieri, 2018).

La muestra está compuesta por 126 estudiantes de la carrera de Derecho de una IES pública de la ciudad de Guayaquil, a los cuales se les formulará un cuestionario bajo la herramienta SERVPERF, con la finalidad de evaluar las dimensiones de esta institución educativa en ámbitos referentes a los elementos tangibles, fiabilidad, capacidad de respuesta, seguridad, empatía y satisfacción.

Para acceder a los datos de la muestra seleccionada, se plantea la utilización de la herramienta SERVPERF, el cual permite medir el grado de satisfacción y el nivel de experiencia de los estudiantes de la carrera de Derecho de una IES pública, mediante la evaluación de cinco dimensiones: elementos tangibles, fiabilidad, capacidad de respuesta, seguridad y empatía. Mediante el proceso investigación se procedió a diseñar, utilizar, validar y aprobar el instrumento de medición.

Para el levantamiento de datos, se realizará una encuesta presentada a través de Google Forms, donde la muestra compuesta por 126 estudiantes de la carrera de Derecho de una IES pública de la ciudad de Guayaquil (mediante el modelo de ecuaciones estructurales se puede realizar estimaciones con pocos registros), los cuales responderán acorde a la situación en que se encuentren en ámbitos referentes a los elementos tangibles, fiabilidad, capacidad de respuesta, seguridad, empatía y satisfacción; con la finalidad de analizar los datos obtenidos y poder formular una respuesta a la problemática planteada.

La técnica para el análisis de los datos otorgados por los 126 estudiantes de la carrera de Derecho de una IES pública de la ciudad de Guayaquil, es el modelo de ecuaciones estructurales. Donde se procedió a realizar un análisis factorial confirmatorio para comprobar la estructura factorial del modelo de los datos obtenidos en la encuesta con la finalidad de probar la influencia de la hipótesis de las dimensiones referente a la calidad del servicio y la satisfacción por medio de ecuaciones estructurales. Además, se realizó un análisis de adecuación de los datos a la técnica de estimación y se descubrió que los datos se distribuyen de manera no anormal.

\section{RESULTADOS}

\section{Estadística descriptiva.}

Los resultados del presente estudio serán evaluados en primera instancia sobre una estadística descriptiva que permitirá observar la percepción que tienen los estudiantes de la carrera de derecho de una IES pública de la ciudad de Guayaquil, con referencia a las variables: Tangibles (T), Fiabilidad (F), Capacidad de respuesta (C), Seguridad (S), Empatía (E) y Satisfacción (SA).

Tabla 2

Media de la variable Tangibles

\begin{tabular}{ll}
\hline Ítem & Valor promedio \\
\hline T1: Equipos modernos y funcionales. & 3,000 \\
T2: Instalaciones cómodas y atractivas & 3,264 \\
T3: Funcionarios presentados adecuadamente. & 3,558 \\
T4: Instalaciones suficientes. & 3,239 \\
\hline
\end{tabular}

Mediante la evaluación de la variable Tangibles, se puede apreciar que la mayoría de estudiantes de la carrera de derecho de una IES pública de la ciudad de Guayaquil, tienen la percepción que las instalaciones no cuentan con equipos modernos y funcionales para brindar un buen servicio, debido a que, de los cuatro ítems analizados, esté ítem T4 $(3,000)$, cuanta con la menor valoración entre los elementos 
encuestados. Por otra parte, el ítem T3 $(3,558)$, que corresponde a que los funcionarios están presentados adecuadamente en sus lugares de trabajo, cuenta con la mejor valoración, haciendo que esté sea un punto destacable en la apreciación del alumnado.

Tabla 3

Media de la variable Fiabilidad

\begin{tabular}{ll}
\hline Ítem & Valor Promedio \\
\hline F1: Interés para solucionar un problema. & 2,834 \\
F2: Soluciones en tiempo prometido. & 2,687 \\
F3: Realización de un buen trabajo. & 3,055 \\
\hline
\end{tabular}

Mediante la evaluación de la variable Fiabilidad, se puede inferir que la mayoría de estudiantes de la carrera de derecho de una IES pública de la ciudad de Guayaquil, tienen la percepción que los funcionarios no dan soluciones a su requerimiento en el tiempo prometido, debido a que, de los tres ítems analizados, esté ítem F2 $(2,834)$, cuanta con la menor valoración entre los elementos encuestados. Por otra parte, el ítem F3 $(3,055)$, que corresponde a que los funcionarios realizan habitualmente bien su trabajo, cuenta con la mejor valoración, haciendo que esté sea un punto destacable en la apreciación del alumnado.

Tabla 4

Media de la variable Capacidad de respuesta

\begin{tabular}{ll}
\hline Ítem & Valor Promedio \\
\hline C1: Información precisa del servicio. & 3,067 \\
C2: Soluciones en un tiempo adecuado. & 3,012 \\
C3: Disposición por ayudar. & 3,196 \\
C4: Respuesta oportuna a solicitudes. & 3,190
\end{tabular}

Mediante la evaluación de la variable Capacidad de respuesta, se puede considerar que la mayoría de estudiantes de la carrera de derecho de una IES pública de la ciudad de Guayaquil, tienen la percepción que los funcionarios no están capacitados para ayudarles a resolver inconvenientes en un tiempo adecuado, debido a que, de los cuatro ítems analizados, esté ítem C2 $(2,834)$, cuanta con la menor valoración entre los elementos encuestados. Por otra parte, el ítem C3 $(3,196)$, que corresponde a que los funcionarios de la siempre están dispuestos a ayudar a sus estudiantes, cuenta con la mejor valoración, haciendo que esté sea un punto destacable en la apreciación del alumnado.
Tabla 5

Media de la variable Seguridad

\begin{tabular}{ll}
\hline Ítem & Valor Promedio \\
\hline S1: Confianza en los procedimientos. & 3,067 \\
S2: Seguridad en la página web. & 3,012 \\
S3: Cordialidad. & 3,196 \\
S4: Apoyo de la institución a funcionarios. & 3,190 \\
S5: Conocimiento suficiente de los funcionarios. & 3,387 \\
S6: Atención personalizada. & 3,080 \\
\hline
\end{tabular}

Mediante la evaluación de la variable Seguridad, se puede apreciar que la mayoría de estudiantes de la carrera de derecho de una IES pública de la ciudad de Guayaquil, tienen la percepción de inseguridad al realizar trámites y requerimientos que realizan a través de la página web, debido a que, de los seis ítems analizados, esté ítem S2 (3,012), cuenta con la menor valoración entre los elementos encuestados. Por otra parte, el ítem S5 $(3,387)$, que corresponde a que los funcionarios tienen conocimiento suficiente para responder a los estudiantes de forma clara y precisa, cuenta con la mejor valoración, haciendo que esté sea un punto destacable en la apreciación del alumnado.

Tabla 6

\begin{tabular}{ll} 
Media de la variable Empatía & \\
\hline Ítem & Valor Promedio \\
\hline E1: Horarios de atención convenientes. & 3,147 \\
E2: Conocimiento de las necesidades. & 2,871 \\
E3: Interés en los requerimientos. & 2,914 \\
\hline
\end{tabular}

Mediante la evaluación de la variable Empatía, se puede inferir que la mayoría de estudiantes de la carrera de derecho de una IES pública de la ciudad de Guayaquil, tienen la percepción de que los funcionarios no conocen las necesidades de sus estudiantes, debido a que, de los tres ítems analizados, esté ítem E2 $(2,871)$, cuanta con la menor valoración entre los elementos encuestados. Por otra parte, el ítem E1 $(3,147)$, que corresponde a que la Carrera de Derecho cuenta con horarios de atención convenientes para atender a sus estudiantes, cuenta con la mejor valoración, haciendo que este sea un punto destacable en la apreciación del alumnado. 
Tabla 7

Media de la variable Satisfacción

\begin{tabular}{ll}
\hline Ítem & Valor Promedio \\
\hline SA1: Satisfacción por estudiar & 3,619 \\
SA2: Disposición por recomendar. & 3,548 \\
SA3: Continuidad de otros estudios. & 3,516 \\
\hline
\end{tabular}

Mediante la evaluación de la variable Satisfacción, se puede considerar que la mayoría de estudiantes de la carrera de derecho de una IES pública de la ciudad de Guayaquil, tienen la percepción de no estar dispuestos a seguir educándose en algunos de los programas (educación continua o postgrado) de la Facultad luego de terminar la carrera que cursa actualmente, debido a que, de los tres ítems analizados, esté ítem SA3 $(3,516)$, cuanta con la menor valoración entre los elementos encuestados. Por otra parte, el ítem SA1 $(3,619)$, que corresponde a que los estudiantes se encuentran satisfechos de manera general, haciendo que esté sea un punto destacable en la apreciación del alumnado.

\section{Análisis Factorial confirmatorio.}

El análisis factorial confirmatorio consiste en determinar la relación entre las variables, donde el indicador mientras sea más cercano a la unidad 1,000 , se entenderá que existe una correcta aplicación de la técnica de investigación (encuesta). Las variables Tangibilidad, Fiabilidad, Seguridad, Empatía y Capacidad de respuesta serán relacionadas con la variable Satisfacción, para proseguir con el desarrollo del estudio.

\section{Análisis convergente.}

Para el análisis convergente se realizó el análisis de Consistencia Interna o Alpha de Cronbach, siguiendo de un análisis de Cargas Factoriales y un análisis de Varianza Extraída Media (Hair et al., 2019).

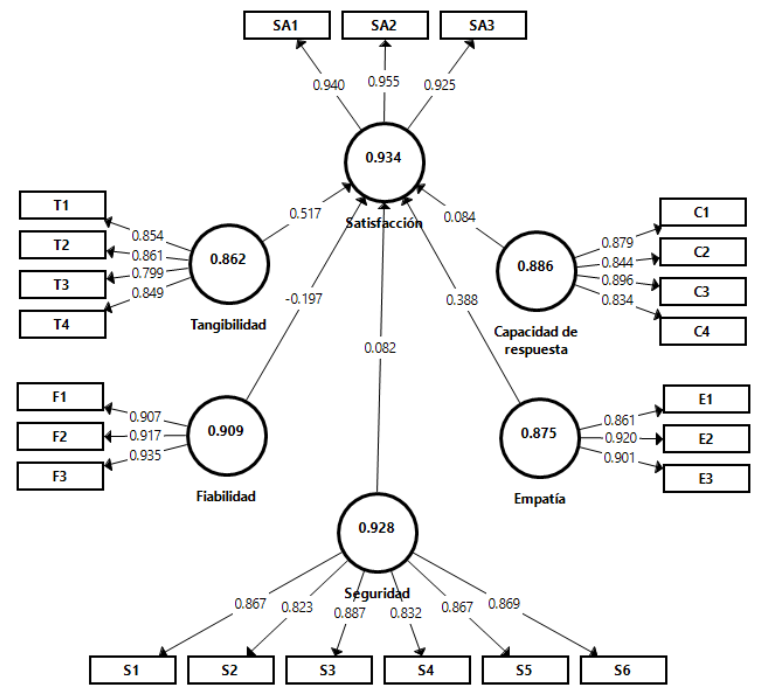

Figura 1: Alfa de Cronbach

Mediante el análisis del Alfa de Cronbach, se establece la siguiente relación: Tangibles 0,862; Fiabilidad 0,909; Seguridad 0,928; Empatía 0,875; Capacidad de respuesta 0,886 y Satisfacción 0,934 .

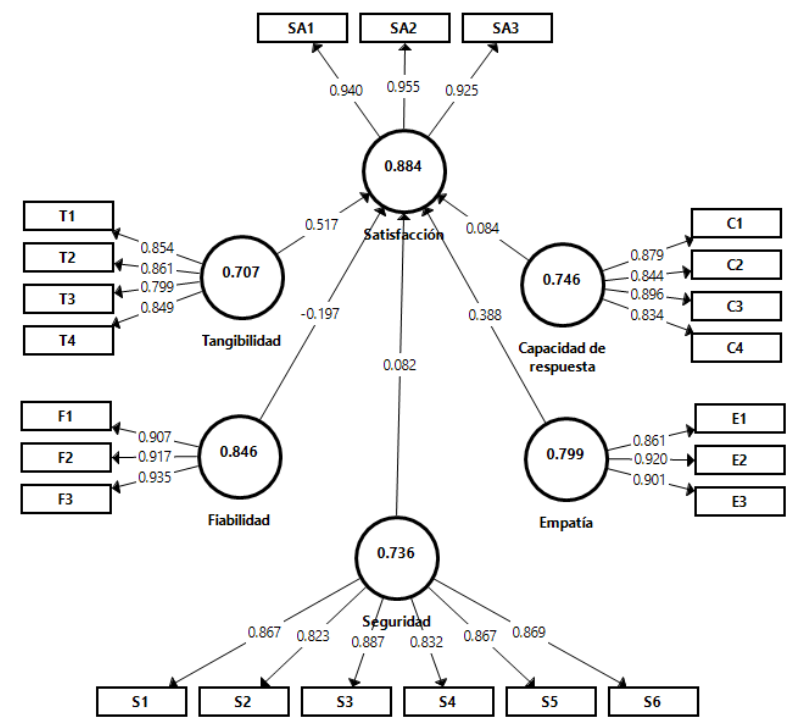

Figura 2: Varianza extraída media

Mediante el análisis de la Varianza extraída media, se establece la siguiente relación: Tangibles 0,707; Fiabilidad 0,846; Seguridad 0,736; Empatía 0,799; Capacidad de respuesta 0,746 y Satisfacción 0,9884 . 


\section{Análisis discriminante.}

Para el análisis discriminante se utilizó el Criterio de Fornell y Larcker y el Criterio de Cargas Cruzadas (Hair et al., 2019).

Tabla 8

Criterio de Fornell y Larcker

\begin{tabular}{lrrrrrr}
\hline & Capacidad de respuesta & Empatía & Fiabilidad & Satisfacción & Seguridad & Tangibilidad \\
\hline Capacidad de respuesta & 0,864 & & & & & \\
Empatía & 0,814 & 0,894 & & & & \\
Fiabilidad & 0,841 & 0,821 & 0,920 & & & \\
Satisfacción & 0,733 & 0,708 & 0,618 & 0,940 & & \\
Seguridad & 0,987 & 0,827 & 0,837 & 0,729 & 0,858 & \\
Tangibilidad & 0,807 & 0,667 & 0,689 & 0,773 & 0,789 & 0,841 \\
\hline
\end{tabular}

Según los valores presentados se cumple con el criterio de Fornell y Larcker y de Cargas cruzadas.

\section{Estimación del modelo estructural.}

El análisis relacional del presente estudio se realizará con dirección a la variable Satisfacción, estableciendo así, los siguientes datos:

\section{Tabla 9}

Estimación del modelo de ecuación estructural

\begin{tabular}{lcc}
\hline Hipótesis & Coeficiente de estimación PLS - SEM & Resultado \\
\hline Tangibilidad $\rightarrow$ Satisfacción servicio & 0.517 & Se acepta \\
Fiabilidad $\rightarrow$ Satisfacción servicio & -0.197 & No se acepta \\
Seguridad $\rightarrow$ Satisfacción servicio & 0.082 & No se acepta \\
Capacidad de Respuesta $\rightarrow$ Satisfacción servicio & 0.084 & No se acepta \\
Empatía $\rightarrow$ Satisfacción servicio & 0.388 & Se acepta \\
\hline
\end{tabular}

Nota: Valor critico de aceptación 0.20 siguiendo el criterio de Hair et al., 2019.

Según los valores otorgados por el análisis relacional, se puede inferir que la variable Tangibles y Empatía tienen una mayor relación hacia la satisfacción, por otra parte, la variable Fiabilidad se encuentra con valor negativo. Por tal motivo desde el punto de vista estratégico es necesario fortalecer las variables con mayor relación a la satisfacción y mejorar las demás variables implicadas.

Dentro de las acciones estratégicas se pueden tomar están las siguientes: (a) Mejorar la página web, (b) Estar en constante innovación de los equipos y evaluar su funcionalidad, (c) Presentar al alumnado un buen cuidado de las instalaciones físicas, (d) Mejorar el tiempo de solución de los requerimientos, (e) Establecer evaluaciones de las necesidades del estudiante de manera constante, (f) Premiar al funcionario por su presentación y rendimiento, (g) Establecer seminarios de atención al cliente dirigido hacia los funcionarios.

Estas medidas son necesarias para que los estudiantes sientan que los procesos de atención que ellos reciben, se realizan con la mayor eficiencia posible. Cabe recalcar que los resultados no son extrapolables hacia otras facultades o instituciones y para una mayor profundización del sistema educativo ecuatoriano son necesarias más investigaciones de esta índole para evaluar la calidad del servicio estudiantil tanto en universidades públicas, como privadas.

\section{CONCLUSIONES}

En el presente estudio se evaluó la calidad del servicio estudiantil en la carrera de Derecho de una Institución de Educación Superior pública de Guayaquil, donde se estableció que la variable Tangibles y Empatía tienen una mayor relación hacia la satisfacción con una relación de 0.517 y 0.388 respectivamente. Por tal motivo desde el punto de vista estratégico es necesario fortalecer las variables con mayor relación a la satisfacción con estrategias vinculadas con el mejoramiento de la página web, establecer un cronograma de innovación de los equipos, mantener un buen cuidado de las instalaciones físicas, optimizar el tiempo de solución de los requerimientos, establecer evaluaciones de las necesidades del estudiante de manera constante, premiar al funcionario por su presentación y rendimiento y brindar seminarios de atención al cliente dirigido hacia los funcionarios.

Cabe recalcar que los resultados no son extrapolables hacia otras facultades o instituciones; lo cual indica que para una mayor profundización de la calidad del servicio del sistema educativo ecuatoriano son necesarias más investigaciones de esta índole, donde intervengan evaluaciones tanto a estudiantes de universidades públicas, como privadas. 


\section{REFERENCIAS}

1. Acosta, A. M., Durán, E. G., \& Padilla, R. P. (2018). Impacto de la calidad de los servicios sobre la satisfacción de los clientes en una empresa de mantenimiento. Redalyc, 21(40): 1-18. https://revistas.uclave.org/index.php/Com pendium/article/view/1656/945.

2. Albrecht, K. (2006). La revolución de servicio (Segunda Edición).

Panamericana Editorial.

3. Alvarado, L. E., Morales, R. D., \& Aguayo, T. E. (2016). Percepción de la calidad educativa: caso aplicado a estudiantes de la Universidad Autónoma de Nuevo León y del Instituto

Tecnológico de Estudios Superiores de Monterrey. Rev. educ. sup, 45(180): 5574.

https://doi.org/10.1016/j.resu.2016.06.00 6.

4. Arancibia, Y. M. (2019). Expectativas y percepción de la calidad de los servicios educativos en los estudiantes de la carrera profesional de administración y negocios internacionales de la Universidad Alas Peruanas - Filial Cajamarca. Perú: Universidad Nacional de Cajamarca.

5. Basco, A. I., Beliz, G., Coatz, D., \& Garnero, P. (2018). Industria 4.0: Fabricando el Futuro. Inter-American Development Bank.

6. Bernal, J., Lauretti, P., \& Agreda, M. (2016). Satisfacción académica en estudiantes de la Facultad de Ingeniería de la Universidad del Zulia. Universidad del Zulia, 301-309.

https://www.redalyc.org/pdf/904/904534 64009.pdf.

7. Boud, D., \& Molloy, E. (2015). El feedback en educación superior y profesional: Comprenderlo y hacerlo bien. Narcea Ediciones.

8. Bracho, M. S., Fernández, M., \& Díaz, J. (2021). Técnicas e instrumentos de recolección de información: análisis y procesamiento realizado por el investigador cualitativo. Uisrael, 8(1): 116.

https://revista.uisrael.edu.ec/index.php/rc ui/article/view/400/197.

9. Brito, L., Quezada, C., \& Arsola, L. (2017). La Universidad y la Vinculación con la Sociedad. Una Condición Impostergable. Editorial UTMACH.

10. Bustamante, C. Z. (Febrero de 2019). Instrumentación de mecanismos para la retención estudiantil en la Universidad del Valle de México en Línea y el Polivirtual . Mexico D.F., Mexico: Centro de Investigación y de Estudios Avanzados del Instituto Politécnico Nacional.

11. Caminotti, M., \& Toppi, H. P. (2020). Metodología de la investigación social: Caja de herramientas. Eudeba.

12. Carmona, C. E. (2020). Hacia la inclusión educativa en la Universidad: Diseño universal para el aprendizaje y la educación de calidad. Ediciones Octaedro.

13. Carrillo, M. I., \& Arroyo, G. C. (2019). Apuntes para caracterizar la formación continua en línea de docentes. Revista de Estudios y Experiencias en Educación, 18(36): 209 - 221.

https://scielo.conicyt.cl/pdf/rexe/v18n36/ 0718-5162-rexe-18-36-209.pdf.

14. Cronin, J., \& Taylor, S. (1992). Measuring service quality: A reexamination and extension. Journal of Marketing, 56(3): 55-68. http://dx.doi.org/10.2307/1252296.

15. Duque, R. O. (2011). Evaluación de la Calidad en Educación Superio. Madrid: Universidad Complutense de Madrid.

16. Farroñan, E. V., García, F. S., Manuel, L. S., \& Morán, I. C. (2020). The Servperf model as a service quality assessment tool in a company. Revista Universidad y Sociedad, 417-423. http://scielo.sld.cu/pdf/rus/v12n2/22183620-rus-12-02-417.pdf. 
17. Galeano-Barrera, C. J., Bellón-Monsalve, D., Zabala-Vargas, S. A., Romero-Riaño, E., \& Duro-Novoa, V. (2018).

Identificación de los pilares que direccionan a una institución universitaria hacia un smart-campus. Rev. Investig.

Desarro. Innov, 9(1),127-145.

http://www.scielo.org.co/pdf/ridi/v9n1/23 89-9417-ridi-9-01-127.pdf.

18. García, A. (2016). Cultura de servicio en la optimización del servicio al cliente.

Universidad Privada Dr. Rafael Belloso Chacín, 381-398.

https://www.redalyc.org/pdf/993/993469 31003.pdf.

19. Gómez, H. O. (2021). COVID-19: acciones para atenuar las emociones negativasdesde los ambientes de aprendizaje. Torreón universitario, 10(28), 1-9.

https://lamjol.info/index.php/torreon/artic le/view/11525/13362.

20. Hair, J. F., MarkoSarstedt, CheahJunHwa, Jan-MichaelBecker, \& M.Ringle, C. (2019). How to specify, estimate, and validate higher-order constructs in PLSSEM. Australian Marketing Journal, 27(3): 197-211.

https://www.sciencedirect.com/science/ar ticle/abs/pii/S1441358219301223.

21. Ibarra, L., \& Casas, E. (2015). Aplicación del modelo Servperf en los centros de atención Telcel, Hermosillo: una medición de la calidad en el servicio. Contaduría y Administración, 60(1): 229260. http://dx.doi.org/10.1016/S01861042(15)72153-4.

22. Jara, E. C. (2017). La calidad del servicio y satisfacción del estudiante dentro de la aplicación de las buenas prácticas del sistema de formación profesional en el Centro Técnico Productiva. Perú: Universidad Cesar Vallejo.

23. Kells, H. (1990). The insufficiency in performance indicators in higher education; the need of the global and formative model. Higher Education Management, 258-270.
24. Lami, J. (2019). Modelo de medición y gestión de la satisfacción del cliente interno y la calidad del servicio financiero en banecuador sucursal Latacunga. Ambato, Ecuador: Universidad Tecnológica Indoamérica .

25. Lewin, L. (2019). Gestión educativa en acción: Cómo desarrollar, motivar y liderar a tu equipo docente. Noveduc.

26. Leyva, R. P. (2017). Academic satisfaction of the nursing studentina public university. Revista Electrónica sobre Tecnología, Educación y Sociedad, 4(7):1-14.

https://www.ctes.org.mx/index.php/ctes/a rticle/view/639/697.

27. López, J. V. (2020). COMT004PO Fundamentos de atención al cliente. Editorial Elearning, S.L.

28. Luyo, V. C., Pachas, B. V., \& Jimenez, A. Y. (2017). Hábitos de estudio, disposición hacia el estudio y rendimiento académico en estudiantes de formación profesional técnica de un instituto armado. Lima, Perú: Universidad Marcelino Champagnat.

29. Matinez, M., Piñero, F., \& Figueroa, S. (2013). El papel de la Universidad en el desarrollo. Benemérita Universidad Autónoma de Puebla.

30. Miranda, P. P., Santiago, Í. C., Atia, V. C., \& Herrera, R. P. (2018). Calidad de Servicio en una institución de educación superior en la ciudad de Barranquilla. Ciencias administrativas, https://revistas.unlp.edu.ar/CADM/article /view/4039.

31. Monje, C. (2011). Metodología de la investigación cuantitativa y cualitativa. Neiva: Universidad Surcolombiana.

32. Moreno, M. C. (2018). Satisfacción estudiantil de la calidad del servicio educativo en la formación profesional de las carreras técnicas de baja y alta demanda. Lima, Perú: Universidad Peruana Cayetano Heredia. 
33. Peñalvo, F. J., Corell, A., Abella-García, V., \& Grande, M. (2020). Online Assessment in Higher Education in the Time of COVID-19. Education in the Knowledge Society, 21(12): 1-26. https://repositorio.grial.eu/bitstream/grial/ 2010/1/a12.pdf.

34. Pérez, A. M., Escoba, C. R., Toledo, M. R., Gutierrez, L. B., \& Reyes, G. M. (2016). Modelo de predicción de la deserción estudiantil de primer año en la Universidad Bernardo O’Higgins. Educ. Pesqui, (44):1-23.

https://www.scielo.br/pdf/ep/v44/15179702-ep-44-e172094.pdf.

35. Péreza, F. L. (2017). Aplicación del modelo SERVPERF para la medición de la percepción sobre la calidad del servicio de la educación superior. Eco Matemático, 43-50.

36. Poveda, G. I. (2020). Evaluación de la calidad de atención y su influencia en la satisfacción de los usuarios de los servicios de salud mental de la zona 8 . Ecuador: Universidad Católica Santiago de Guayaquil.

37. Reinoso, A. J. (2015). expectativa y satisfacción estudiantil por el servicio académico de la universidad privada de tacna. Revista veritas et scientia - UPT, 16-26.

http://revistas.upt.edu.pe/ojs/index.php/ve stsc/article/view/211/195.

38. Rojas, J. C., Mesa, A. P., \& Basulto, M. S. (2018). Enfoques teóricos para la evaluación de la eficiencia y eficacia en el primer nivel de atención médica de los servicios de salud del sector público. Rev retos, 12(1): 96-118.

http://scielo.sld.cu/pdf/rdir/v12n1/rdir061 18.pdf.

39. Sampieri, R. H. (2018). Metodología de la investigación: las rutas cuantitativa, cualitativa y mixta. McGraw-Hill Interamericana.

40. Sánchez, N. G. (2018). Calidad del servicio administrativo y satisfacción modelo q+4d del estudiante de la Universidad Nacional de Huancavelica. Perú: Universidad Nacional de Huancavelica.

41. Taquez, H., Rengifo, D., \& Mejía, D. (2017). Diseño de un instrumento para evaluar el nivel de uso y apropiación de las TIC en una institución de educación superior. Reposital, 1-30. https://reposital.cuaieed.unam.mx:8443/x mlui/handle/20.500.12579/5019.

42. Tipa, J. (2018). ¿De qué me sirve la interculturalidad? Evaluación de la Universidad Intercultural de Chiapas por sus estudiantes. Alteridad, 13(1): 56-71. http://scielo.senescyt.gob.ec/pdf/alteridad /v13n1/1390-325X-Alteridad-13-0100056.pdf.

43. Torres, M. (15 de Diciembre de 2019). La deserción universitaria en el país alcanza el $26 \%$. Diario Expreso, págs. https://www.expreso.ec/guayaquil/deserci on-universitaria-pais-alcanza-261456.html.

44. Torres, Y. E. (2018). Calidad educativa y gestión escolar. Dialnet, 21(2): 259-281. https://dialnet.unirioja.es/servlet/articulo? codigo $=6718920$.

45. Verdesoto, G. J., Mora, K. G., \& Torres, L. G. (2018). análisis de la deserción estudiantil en las universidades del Ecuador y América Latina. Revista Pertinencia Académica, 1-28. http://revistaacademica.utb.edu.ec/index.php/pertacad e/article/view/127/91.

46. Vergara, J. C., Quesada, V. M., \& Maza, F. J. (2014). Effect of the demographic aspects in the valuation of the quality of the service: a review.

Rev.udcaactual.divulg.cient, 20(2): 457467.

http://www.scielo.org.co/scielo.php?pid= S012342262017000200023\&script=sci_abstrac t\&tlng=en. 
47. Vicent, M., Gonzálvez, C., Sanmartín, R., \& Martínez-Monteagudo, M. C. (2018).

Evaluación de la calidad de las estrategias metodológicas empleadas en la docencia universitaria: satisfacción del alumnado del Grado de Maestro. Octaedro S.L.

48. Vizcaíno, A. d., Luis, A. L., \& Rocha, B. P. (2017). Gestión del conocimiento desde el modelo servperf: un estudio de la calidad del servicio en una comercializadora. Memoria del XI Congreso de la Red Internacional de Investigadores en Competitividad, 13771395.

https://riico.net/index.php/riico/article/vie w/1508/1168.

49. Vizcaíno, A., Marín, V. V., \& Vargas, J. (2016). Evaluación del servicio de urgencias en un hospital público a través del modelo Servperf. Memoria del X
Congreso de la Red Internacional de Investigadores en competitividad, 530543.

https://riico.net/index.php/riico/article/vie w/1332/1001.

50. Zacarías, H., \& Supo, J. (2020). MetodologÍa de la Investigación Científica: Para Las Ciencias de la Salud y Las Ciencias Sociales. Independently Published.

51. Zamora, W. M., Mieles, T. J., Ponce, E. A., \& Ponce, S. X. (2019). La calidad total como fuente de ventaja competitiva en las pequeñas y medianas empresas (Pymes) del Ecuador. Revista Científica de Investigación actualización del mundo de las Ciencias, 3(1): 963-984. https://www.reciamuc.com/index.php/RE CIAMUC/article/view/295/311. 